\section{CRISPR screen for metastasis}

The genome-editing technology derived from the bacterial CRISPR/Cas9 system is revolutionizing biomedical research. Using a CRISPR-based strategy, Feng Zhang, Phillip Sharp and colleagues performed a genome-wide loss-of-function genetic screen in vivo, uncovering new genes involved in tumor growth and metastasis (Cell doi:10.1016/j.cell.2015.02.038; 5 March 2015). They started by transducing a non-metastatic lung cancer cell line with a library of 67,405 single-guide RNAs (sgRNAs). When transplanted into immunocompromised mice, the mutated cell pool readily generated metastases in the lung. These tumors were then analyzed to look for gene-specific sgRNA enrichment. The list of top candidates includes known tumor-suppressor genes but also genes with no previously described role in tumor development. This work advances the understanding of cancer evolution and elegantly underscores the potential of CRISPR/Cas9-based screens to study biological phenomena in vivo. It will be interesting to conduct similar cancer studies in other organs, potentially using gain-of-function approaches to identity novel oncogenes.

\section{The two sides of GIGANTEA}

The Arabidopsis thaliana late-flowering mutation gigantea (GI) alters the circadian period and abiotic stress resistance. Now, C. Robertson McClung and colleagues report the fine mapping and cloning of the causal polymorphism in the Brassica rapa GI locus using recombinant inbred lines and show that it is responsible for variation in cold and salt tolerance (Proc. Natl. Acad. Sci. USA 112, 905-910, 2015). Null alleles for this gene were then identified among B. rapa TILLING mutants, and the authors show that these plants were arrhythmic or had abnormally long circadian periods in response to high temperature, as in Arabidopsis. The B. rapa alleles from the two parental strains could both rescue the photoperiodic flowering defect of the Arabidopsis gi null mutant. However, only one of the two B. rapa alleles was able to rescue the mutant phenotypes of increased freezing tolerance, decreased nitrogen accumulation and increased salt resistance. These results indicate that the effect of GI on flowering time is independent from its role in abiotic stress resistance and that these functions can be separated at the genetic level, which may have practical implications for targeted breeding programs in Brassica crops.

\section{Epigenetic evolution of corticogenesis}

Human brains have a uniquely large and functionally complex neocortex. To understand the features of cortical development that are unique to humans, James Noonan and colleagues mapped active promoters and enhancers during human, rhesus macaque and mouse corticogenesis (Science 347, 1155-1159, 2015). The authors profiled $\mathrm{H} 3 \mathrm{~K} 27 \mathrm{ac}$ and $\mathrm{H} 3 \mathrm{~K} 4 \mathrm{me} 2$ histone marks in cortical tissues at multiple homologous stages of corticogenesis to map active promoters and enhancers. They identified 8,996 enhancers and 2,855 promoters that

Written by Tiago Faial, Brooke LaFlamme, Emily Niemitz \& Kyle Vogan had an increase in $\mathrm{H} 3 \mathrm{~K} 27 \mathrm{ac}$ or H3K4me2 levels in human tissues compared to rhesus macaque and mouse tissues. Using available corticogenesis expression data, they generated a network model of cortical development and identified 96 sets of genes with highly correlated expression (modules). They integrated the lists of enhancers and promoters with human increases in $\mathrm{H} 3 \mathrm{~K} 27 \mathrm{ac}$ or $\mathrm{H} 3 \mathrm{~K} 4 \mathrm{me} 2$ signals and identified 17 modules that were enriched for these signals. These modules include genes associated with cortical development, neuronal progenitor proliferation, the extracellular matrix, and TGF- $\beta$ and FGF signaling.

EN

\section{ALS susceptibility genes}

Tim Harris and colleagues (Science doi:10.1126/science.aaa3650; 19 February 2015) report the results of an exome sequencing study designed to identify new susceptibility genes for amyotrophic lateral sclerosis (ALS). The authors sequenced and analyzed the exomes of 2,874 ALS cases and 6,405 controls, with follow-up analyses of 51 selected genes in an additional 1,318 ALS cases and 2,371 controls. In gene-based burden tests comparing cases with controls, they observed a genome-wide significant excess of rare variants in SOD1, a known ALS susceptibility gene, and in TBK1, a gene not previously implicated in disease risk. They also found supportive evidence for several previously reported ALS susceptibility genes in their data set, including TARDBP, OPTN, VCP and SPG11. TBK1 encodes a kinase that functions in the NF- $\kappa B$ pathway and that phosphorylates several proteins in the autophagy pathway, including OPTN. Additional studies will be needed to clarify the molecular mechanisms by which TBK1 variants contribute to disease risk. The work also suggests that ongoing sequencing studies in larger collections of ALS cases and controls will yield additional insights into the genes and pathways influencing ALS pathogenesis.

KV

\section{Mouse modeling of Alzheimer's disease}

Most cases of familial Alzheimer's disease are caused by mutations in PSEN1 (encoding presenilin-1). However, whether these missense mutations function through a loss- or gain-offunction mechanism is unclear. Previously, a gain-of-function mechanism was proposed on the basis of increased A $\beta 42 /$ A 440 ratios in patients with PSEN1-mutant familial Alzheimer's disease. Now, Raymond Kelleher III, Jie Shen and colleagues report that Psen 1 knock-in mice generated with either of two missense mutations from familial Alzheimer's disease show complete loss of presenilin- 1 function due to loss of catalytic activity, confirming earlier in vitro results (Neuron 85, 967-981, 2015). The phenotypes of homozygous knock-in mice were indistinguishable from those of Psen1-null mice: perinatal lethality, developmental defects and impaired neurogenesis due to disruption of Notch signaling. Heterozygous mutants developed normally but had reduced $A \beta$ production and increased $A \beta 42 /$ A $\beta 40$ ratios by 3 months of age and showed Alzheimer's diseaselike age-dependent neurodegeneration and memory impairment. These results suggest that restoration of normal presenilin-1 function might be a promising therapeutic strategy for some patients with Alzheimer's disease. 